\title{
ALGEBRAIC METHODS IN APPROXIMATION THEORY
}

\author{
HAL SCHENCK
}

\begin{abstract}
This survey gives an overview of several fundamental algebraic constructions which arise in the study of splines. Splines play a key role in approximation theory, geometric modeling, and numerical analysis; their properties depend on combinatorics, topology, and geometry of a simplicial or polyhedral subdivision of a region in $\mathbb{R}^{k}$, and are often quite subtle. We describe four algebraic techniques which are useful in the study of splines: homology, graded algebra, localization, and inverse systems. Our goal is to give a hands-on introduction to the methods, and illustrate them with concrete examples in the context of splines. We highlight progress made with these methods, such as a formula for the third coefficient of the polynomial giving the dimension of the spline space in high degree. The objects appearing here may be computed using the spline package of the Macaulay2 software system.
\end{abstract}

\section{Contents}

1. Introduction

2. Homology and chain complexes

2.1. Algebraic setting

2.2. Topological motivation

2.3. Splines and homology

3. Graded algebra

3.1. Rings and modules, Hilbert polynomial and series 8

3.2. Free resolutions

3.3. Grading in the spline setting

4. Localization

4.1. Basics of the construction

4.2. Application: polyhedral splines 10

4.3. Application: vanishing of homology 16

5. Inverse systems and powers of linear forms $\quad 17$

5.1. Powers of linear forms and fatpoints 18

5.2. Application: planar splines of mixed smoothness 18

$\begin{array}{ll}\text { 5.3. A conjecture in algebraic geometry } & 19\end{array}$

6. Open questions 20

6.1. Higher dimensions 20

6.2. Polyhedral complexes $\quad 21$

6.3. Supersmoothness 21

References $\quad 21$

2000 Mathematics Subject Classification. Primary 41A15, Secondary 13D40, 14M25, 55N30. Key words and phrases. spline, polyhedral complex, homology, localization, inverse system. Schenck supported by NSF 1312071. 


\section{INTRODUCTION}

In mathematics it is often useful to approximate a function $f$ on a region by a simpler function. A natural way to do this is to divide the region into simplices or polyhedra, and then approximate $f$ on each simplex by a polynomial function. A $C^{r}$-differentiable piecewise polynomial function on a $k$-dimensional simplicial or polyhedral subdivision $\Delta \subseteq \mathbb{R}^{k}$ is called a spline. Splines are ubiquitous in geometric modeling and approximation theory, and play a key role in the finite element method for solving PDE's. There is also a great deal of beautiful mathematical structure to these problems, involving commutative and homological algebra, geometry, combinatorics and topology.

For a fixed $\Delta$ and choice of smoothness $r$, the set of splines where each polynomial has degree at most $d$ is a real vector space, denoted $S_{d}^{r}(\Delta)$. The dimension of $S_{d}^{r}(\Delta)$ depends on $r, d$ and the geometric, combinatorial, and topological properties of $\Delta$. For many important cases, there is no explicit general formula known for this dimension. In applications, it will also be important to find a good basis, or at least a good generating set for $S_{d}^{r}(\Delta)$; in this context good typically means splines which have a small support set.

Splines seem to have first appeared in a paper of Courant [12], who considered the $C^{0}$ case. Pioneering work by Schumaker [41] in the planar setting established a dimension formula for all $d$ when $\Delta$ has a unique interior vertex, as well as a lower bound for any $\Delta$ :

Theorem 1.1. [Schumaker, [41]] For a simplicial complex $\Delta \subseteq \mathbb{R}^{2}$

$$
\operatorname{dim} S_{d}^{r}(\Delta) \geq\left(\begin{array}{c}
d+2 \\
2
\end{array}\right)+\left(\begin{array}{c}
d-r+1 \\
2
\end{array}\right) f_{1}^{0}-\left(\left(\begin{array}{c}
d+2 \\
2
\end{array}\right)-\left(\begin{array}{c}
r+2 \\
2
\end{array}\right)\right) f_{0}^{0}+\sum \sigma_{i}
$$

where $f_{1}^{0}=\mid$ interior edges $\left|, f_{0}^{0}=\right|$ interior vertices $\mid$, and $\sigma_{i}=\sum_{j} \max \{(r+1+$ $\left.\left.j\left(1-n\left(v_{i}\right)\right)\right), 0\right\}$, with $n\left(v_{i}\right)$ the number of distinct slopes at an interior vertex $v_{i}$.

Using Bezier-Bernstein techniques, Alfeld-Schumaker prove in [4] that if $d \geq 4 r+$ 1 then equality holds in Theorem 1.1. Hong [23] shows equality holds if $d \geq 3 r+2$, and Alfeld-Schumaker show in [5] equality holds for $d \geq 3 r+1$ and $\Delta$ generic. There remain tantalizing open questions in the planar case: the Oberwolfach problem book from May 1997 contains a conjecture of Alfeld-Manni that for $r=1$ Theorem 1.1 gives the dimension in degree $d=3$. Work of Diener [15] and Tohaneanu [45] shows the next conjecture is optimal.

Conjecture 1.2. [32] The Schumaker formula holds with equality if $d \geq 2 r+1$.

Homological methods were introduced to the field in a watershed 1988 paper of Billera [8], which solved a conjecture of Strang [44] on the dimension of $S_{2}^{1}(\Delta)$ for a generic planar triangulation. One key ingredient in the work was a result of Whiteley [46] using rigidity theory. Homological methods are discussed in detail in $\S 2$, and the utility of these tools is illustrated in $\S 4$.

A useful observation is that the smoothness condition is local: for two $k$ simplices $\sigma_{1}$ and $\sigma_{2}$ sharing a common $k-1$ face $\tau$, let $l_{\tau}$ be a nonzero linear form vanishing on $\tau$. Then a pair of polynomials $f_{1}, f_{2}$ meet with order $r$ smoothness across $\tau$ iff $l_{\tau}^{r+1} \mid f_{1}-f_{2}$. For splines on a line, the situation is easy to understand, so the history of the subject really begins with the planar case. Even the simplest case is quite interesting: let $\Delta \subseteq \mathbb{R}^{2}$ be the star of a vertex, so that $\Delta$ is triangulated with a single interior vertex as in the next example. 
Example 1.3. A planar $\Delta$ which is the star of a single interior vertex $v_{0}$ at the origin.

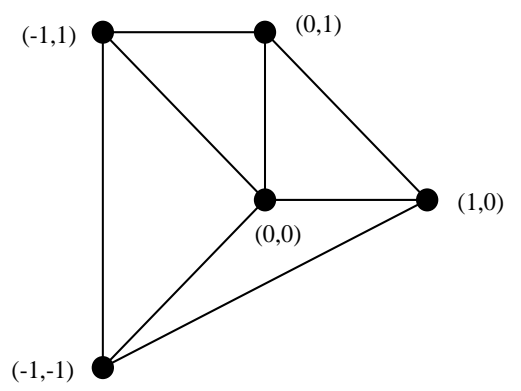

Starting with the triangle in the first quadrant and moving clockwise, label the polynomials on the triangles $f_{1}, \ldots, f_{4}$. To obtain a global $C^{r}$ function, we require

$$
\begin{array}{cc}
a_{1} y^{r+1} & =f_{1}-f_{2} \\
a_{2}(x-y)^{r+1} & =f_{2}-f_{3} \\
a_{3}(x+y)^{r+1} & =f_{3}-f_{4} \\
a_{4} x^{r+1} & =f_{4}-f_{1}
\end{array}
$$

Summing both sides yields the equation $\sum_{i=1}^{4} a_{i} l_{i}^{r+1}=0$ (where $l_{1}=y$ and so on), and gives a hint that algebra has a role to play. $\diamond$

Definition 1.4. Let $\left\{f_{1}, \ldots, f_{m}\right\}$ be a set of polynomials. A syzygy is a relation

$$
\sum_{i=1}^{m} a_{i} f_{i}=0, \text { where the } a_{i} \text { are also polynomials. }
$$

Notice that if each $f_{i}$ is a fixed polynomial $f$, then the smoothness condition is trivially satisfied.

Definition 1.5. For any $r, d, \Delta$, the set of polynomials of degree at most $d$ is a subspace of $S_{d}^{r}(\Delta)$, which we call global polynomials.

Using the vector space structure and the global polynomials, we may assume $f_{1}=0$. This means that given a syzygy on

$$
\left\{y^{r+1},(x-y)^{r+1},(x+y)^{r+1}, x^{r+1}\right\},
$$

we can reverse the process and solve for the $f_{i}$. So when $\Delta=\operatorname{st}(\mathrm{v}) \subseteq \mathbb{R}^{2}$ is triangulated with a single interior vertex as in the example above, $S_{d}^{r}(\operatorname{st}(\mathrm{v}))$ consists of global polynomials and syzygies. To actually compute the dimension and basis for the space of syzygies is nontrivial:

Theorem 1.6. [Schumaker, [41]] For a planar simplicial complex $\Delta=\operatorname{st}(\mathrm{v})$ with $f_{1}^{0}$ interior edges and $n$ distinct slopes at the interior vertex $v$,

$$
\begin{array}{ccc}
\operatorname{dim} S_{d}^{r}(\mathrm{st}(\mathrm{v})) & = & \left(\begin{array}{c}
d+2 \\
2
\end{array}\right) \\
+ & \left(\begin{array}{c}
d-r+1 \\
2
\end{array}\right) f_{1}^{0} \\
& - & \left(\left(\begin{array}{c}
d+2 \\
2
\end{array}\right)-\left(\begin{array}{c}
r+2 \\
2
\end{array}\right)\right) \\
+ & \sum_{j \geq 0} \max \{(r+1+j(1-n)), 0\}
\end{array}
$$


Throughout this paper, $\Delta \subseteq \mathbb{R}^{k}$ is a simplicial or polyhedral complex; $\Delta_{i}$ and $\Delta_{i}^{0}$ denote the sets of $i$-dimensional faces and $i$-dimensional interior faces; $f_{i}(\Delta)=\left|\Delta_{i}\right|$ and $f_{i}^{0}(\Delta)=\left|\Delta_{i}^{0}\right|$. Finally, $\Delta$ is a pseudomanifold: every $k-1$ simplex $\tau \notin \partial(\Delta)$ is a face of exactly two $k$-simplices, and for any pair $\sigma, \sigma^{\prime} \in \Delta_{k}$, there is a sequence $\sigma=\sigma_{1}, \ldots \sigma_{m}=\sigma^{\prime}$ with $\sigma_{i} \cap \sigma_{i+1} \in \Delta_{k-1}$. Our references are de Boor [14] and Lai-Schumaker [25] for splines and Eisenbud [19] and Schenck [34] for algebra.

\section{Homology and Chain COMPLEXES}

In this section, we give an overview of the methods introduced in the study of splines by Billera in [8]. Subsequent progress using homological methods appears in Billera-Rose [9], [10], Yuzvinsky [47], Schenck-Stillman [39], [40], Rose [30],[31], Mourrain-Villamizar [29], DiPasquale [16], [17], and more. We begin with a review of the algebraic and topological basics, and then specialize to the case of splines.

2.1. Algebraic setting. A sequence of vector spaces and linear transformations

$$
\mathcal{C}: \quad \cdots \stackrel{\phi_{j+2}}{\longrightarrow} V_{j+1} \stackrel{\phi_{j+1}}{\longrightarrow} V_{j} \stackrel{\phi_{j}}{\longrightarrow} V_{j-1} \stackrel{\phi_{j-1}}{\longrightarrow} \cdots
$$

is called a complex (or chain complex) if

$$
\operatorname{im}\left(\phi_{j+1}\right) \subseteq \operatorname{ker}\left(\phi_{j}\right) .
$$

The sequence is exact at position $j$ if $\operatorname{im}\left(\phi_{j+1}\right)=\operatorname{ker}\left(\phi_{j}\right)$; a complex which is exact everywhere is called an exact sequence. We define the homology of the complex $\mathcal{C}$ as

$$
H_{j}(\mathcal{C})=\operatorname{ker}\left(\phi_{j}\right) / \operatorname{im}\left(\phi_{j+1}\right) .
$$

Example 2.1. Consider the complex

$$
0 \longrightarrow V_{1} \stackrel{\phi}{\longrightarrow} V_{0} \longrightarrow 0
$$

where $V_{1}=V_{0}=\mathbb{R}^{3}$ and $\phi$ is:

$$
\left[\begin{array}{ccc}
-1 & 0 & 1 \\
1 & -1 & 0 \\
0 & 1 & -1
\end{array}\right]
$$

$H_{1}(\mathcal{C})=\operatorname{ker}(\phi)$ has basis $[1,1,1]^{t}$ and $H_{0}(\mathcal{C})=\operatorname{coker}(\phi)=\mathbb{R}^{3} / \operatorname{im}(\phi) . \diamond$

For a complex of finite dimensional vector spaces

$$
\mathcal{C}: 0 \longrightarrow V_{n} \longrightarrow V_{n-1} \longrightarrow \cdots \longrightarrow V_{1} \longrightarrow V_{0} \longrightarrow 0
$$

the alternating sum of the dimensions is called the Euler characteristic of $\mathcal{C}$, and written $\chi(\mathcal{C})$; when $\mathcal{C}$ is exact $\chi(\mathcal{C})=0$, and an easy induction shows that in general

$$
\chi(\mathcal{C})=\sum_{i=0}^{n}(-1)^{i} \operatorname{dim} V_{i}=\sum_{i=0}^{n}(-1)^{i} \operatorname{dim} H_{i}(\mathcal{C}) .
$$

Everything defined above generalizes in the obvious way to sequences of modules and homomorphisms. 
Definition 2.2. A short exact sequence of complexes is a commuting diagram:

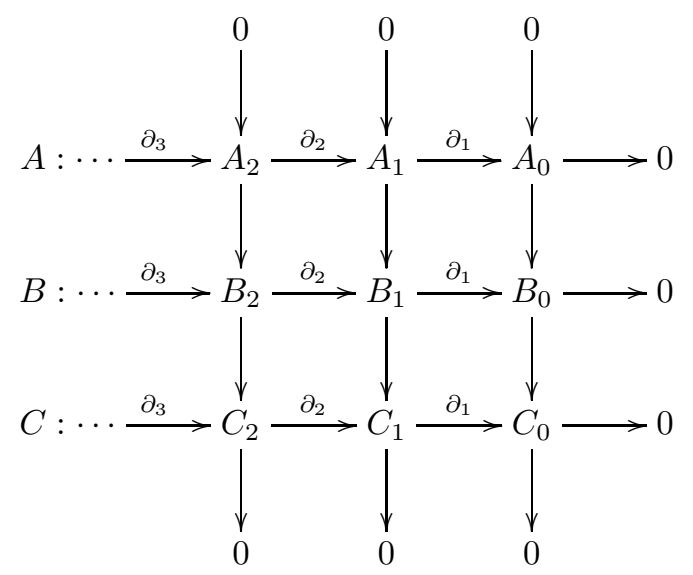

where the columns are exact and the rows are complexes.

Theorem 2.3. A short exact sequence of complexes yields a long exact sequence in homology:

$$
\cdots \longrightarrow H_{n+1}(C) \longrightarrow H_{n}(A) \longrightarrow H_{n}(B) \longrightarrow H_{n}(C) \longrightarrow H_{n-1}(A) \longrightarrow \cdots
$$

For additional details and proof, see Chapter 8 of [34].

2.2. Topological motivation. The invention of homology has topological roots. The basic idea is to model topological objects with combinatorial ones; in particular there is no topological difference between the circle and the boundary of a triangle, or between the sphere and the boundary of a tetrahedron. This generalizes, and the idea is to approximate a topological space $X$ by using simplices, which are higher dimensional analogs of triangles. A union of simplices forms a simplicial complex $\Delta$, giving a combinatorial approximation to $X$. The data of $\Delta$ allows us to build an algebraic chain complex $\mathcal{C}(\Delta)$, whose homology encodes topological information.

An abstract $n$-simplex is a set consisting of all subsets of an $n+1$ element ground set. Typically a simplex is viewed as a geometric object; for example a twosimplex on the set $\{a, b, c\}$ can be visualized as a triangle, with the subset $\{a, b, c\}$ corresponding to the whole triangle, $\{a, b\}$ an edge, and $\{a\}$ a vertex.

Definition 2.4. [Chapter 5, [34]] A simplicial complex $\Delta$ on a vertex set $V$ is a collection of subsets $\gamma$ of $V$, such that if $\gamma \in \Delta$ and $\tau \subseteq \gamma$, then $\tau \in \Delta$. If $|\gamma|=i+1$ then $\gamma$ is called an $i$-face. An oriented simplex is a simplex with a fixed ordering of the vertices, modulo an equivalence relation: for a permutation $\sigma \in S_{n}$ and oriented simplex $\tau=\left[i_{1}, \ldots, i_{n}\right], \tau \sim(-1)^{\operatorname{sgn}(\sigma)} \sigma(\tau)$.

Example 2.5. Label the vertices in Example 1.3 as $v_{0}=(0,0), v_{1}=(1,0), v_{2}=$ $(-1,-1), v_{3}=(-1,1), v_{4}=(0,1)$. Then the set of four oriented triangles

$$
\left[v_{0}, v_{1}, v_{4}\right],\left[v_{0}, v_{2}, v_{1}\right],\left[v_{0}, v_{3}, v_{2}\right],\left[v_{0}, v_{4}, v_{3}\right]
$$

eight oriented edges

$$
\left[v_{0}, v_{1}\right],\left[v_{0}, v_{2}\right],\left[v_{0}, v_{3}\right],\left[v_{0}, v_{4}\right],\left[v_{1}, v_{2}\right],\left[v_{2}, v_{3}\right],\left[v_{3}, v_{4}\right],\left[v_{4}, v_{1}\right]
$$

and vertices $\left[v_{0}\right], \ldots,\left[v_{4}\right]$ form an oriented simplicial complex. $\diamond$ 
Definition 2.6. Let $C_{i}(\Delta)$ be a free $R$-module with basis the oriented $i$-simplices, and define a map $C_{i}(\Delta) \stackrel{\partial_{i}}{\longrightarrow} C_{i-1}(\Delta)$ via

$$
\partial_{i}\left[e_{j_{0}}, \ldots e_{j_{i}}\right]=\sum_{m=0}^{i}(-1)^{m}\left[e_{j_{0}}, \ldots \widehat{e_{j_{m}}}, \ldots e_{j_{i}}\right]
$$

A check shows that $\partial_{i} \circ \partial_{i+1}=0$, hence $(C(\Delta), \partial)$ is a chain complex. The homology of $(C(\Delta), \partial)$ encodes topological information. If $\Delta=$ $\left\{\left[v_{0}\right],\left[v_{1}\right],\left[v_{2}\right],\left[v_{0} v_{1}\right],\left[v_{1} v_{2}\right],\left[v_{2} v_{0}\right]\right\}$, then $\Delta \sim S^{1}$ and $C(\Delta)$ is the complex of Example 2.1. $H_{1}(\Delta) \sim \mathbb{R}$ captures the fact that $S^{1}$ is not simply connected.

2.3. Splines and homology. The compatibility condition discussed in $\S 1$ has a beautiful interpretation in terms of homology: suppose $\Delta \subseteq \mathbb{R}^{k}$ and $\sigma$ and $\sigma^{\prime} \in \Delta_{k}$ satisfy

$$
\sigma \cap \sigma^{\prime}=\tau \in \Delta_{k-1} .
$$

Then if $f$ is a polynomial on $\sigma$ and $f^{\prime}$ a polynomial on $\sigma^{\prime}$, the set of pairs $\left(f, f^{\prime}\right)$ which glue $C^{r}$ smoothly across $\tau$ is the kernel of the map

$$
R^{2} \stackrel{[1,-1]}{\longrightarrow} R / l_{\tau}^{r+1} .
$$

Example 2.7. In relative homology, the modules $C_{i}(\Delta)$ are quotiented by chains $C_{i}\left(\Delta^{\prime}\right)$ of a subcomplex $\Delta^{\prime}$. In the spline setting, the subcomplex is $\partial(\Delta)$. For Example 2.5, applying Definition 2.6 for the relative complex yields

$$
\partial_{2}=\left[\begin{array}{cccc}
1 & -1 & 0 & 0 \\
0 & 1 & -1 & 0 \\
0 & 0 & 1 & -1 \\
-1 & 0 & 0 & 1
\end{array}\right]
$$

We compute

$$
\partial_{2}\left[f_{1}, f_{2}, f_{3}, f_{4}\right]^{t}=\left[\begin{array}{c}
f_{1}-f_{2} \\
f_{2}-f_{3} \\
f_{3}-f_{4} \\
f_{4}-f_{1}
\end{array}\right]
$$

This is the right hand side of Equation $1 . \diamond$

We still need to encode the smoothness condition, and Equation 3 provides the clue: rather than having $\partial_{2}$ map a free module to another free module, we enrich our chain complex to include the smoothness condition:

Example 2.8. Continuing with the previous example, define a map

$$
\bigoplus_{\sigma \in \Delta_{2}} R \stackrel{\partial_{2}}{\longrightarrow} \bigoplus_{\tau \in \Delta_{1}^{0}} R / l_{\tau}^{r+1}
$$

The kernel of this map consists exactly of polynomial vectors $\left[f_{1}, f_{2}, f_{3}, f_{4}\right]$ which satisfy the conditions of Equation 1. This suggests how to define a chain complex whose top homology module consists of splines on $\Delta$. $\diamond$

Example 2.9. Let $\mathcal{R}$ be the constant complex on $\Delta^{0}: \mathcal{R}(\sigma)=R$, for every $\sigma \in \Delta^{0}$. Take $\partial_{i}$ to be the usual (relative to $\partial \Delta$ ) simplicial boundary map. Then $H_{i}(\mathcal{R})$ is the usual (modulo boundary) simplicial homology, with coefficients in $R$. $\diamond$ 
Example 2.10. In [8], Billera defined the following complex: for each $\sigma \in \Delta^{0}$, let $I_{\sigma}$ be the ideal of $\sigma \subset \mathbb{R}^{k}$. $I_{\sigma}$ is generated by linear polynomials. Fix $r \in \mathbb{N}$, and define a complex $\mathcal{I}$ of ideals on $\Delta$ by $\mathcal{I}(\sigma)=I_{\sigma}^{r+1}$, and define the quotient complex $\mathcal{R} / \mathcal{I}$ via $\mathcal{R} / \mathcal{I}(\sigma)=R / I_{\sigma}^{r+1}$. $\diamond$

Example 2.11. The paper [39] modifies Billera's complex. Fix $r \in \mathbb{N}$, and define a complex $\mathcal{J}$ of ideals on $\Delta$ by

$$
J_{\psi}=\left\langle l_{\tau_{1}}^{r+1}, \ldots, l_{\tau_{n}}^{r+1} \mid \psi \in \tau_{i} \in \Delta_{d-1}^{0}\right\rangle .
$$

Define the quotient complex $\mathcal{R} / \mathcal{J}$ via $\mathcal{R} / \mathcal{J}(\psi)=R / J_{\psi}$. $\diamond$

While the complexes $\mathcal{R} / \mathcal{I}$ and $\mathcal{R} / \mathcal{J}$ agree at positions $k$ and $k-1$, they typically differ in lower degrees. Since $\mathcal{J}$ is a submodule of $\mathcal{R}$, the differential in the complex of Example 2.9 induces a differential on $\mathcal{J}$ and on $\mathcal{R} / \mathcal{J}$. By Theorem 2.3, the short exact sequence of complexes

$$
0 \longrightarrow \mathcal{J} \longrightarrow \mathcal{R} \longrightarrow \mathcal{R} / \mathcal{J} \longrightarrow 0
$$

gives rise to a long exact sequence in homology:

$$
\longrightarrow H_{i+1}(\mathcal{R} / \mathcal{J}) \longrightarrow H_{i}(\mathcal{J}) \longrightarrow H_{i}(\mathcal{R}) \longrightarrow H_{i}(\mathcal{R} / \mathcal{J}) \longrightarrow H_{i-1}(\mathcal{J}) \longrightarrow \cdots
$$

Billera showed $H_{k}(\mathcal{R} / \mathcal{I})_{d}=S_{d}^{r}(\Delta)$. Since $\mathcal{J}$ and $\mathcal{I}$ agree on the $k$ and $k-1$ faces,

$$
H_{k}(\mathcal{R} / \mathcal{J})_{d}=H_{k}(\mathcal{R} / \mathcal{I})_{d}=S_{d}^{r}(\Delta) .
$$

However, the lower homology modules differ; in the complex $\mathcal{R} / \mathcal{J}$, information is more evenly balanced between the homology modules and the modules of the chain complex: neither of these sets of modules is simple to understand. On the other hand, the modules in the chain complex $\mathcal{R} / \mathcal{I}$ are easy to understand, so that for the chain complex $\mathcal{R} / \mathcal{I}$, all geometric information is encoded in the homology modules, making them difficult to decipher. In $\S 4$, we use localization to prove

Theorem 2.12 ([33]). If $\Delta \subseteq \mathbb{R}^{k}$ simplicial, then for all $i<k$ and $d \gg 0$, $\operatorname{dim}_{\mathbb{R}} H_{i}(\mathcal{R} / \mathcal{J})_{d}$ is given by a polynomial in $d$ of degree at most $i-2$.

For $\Delta \subseteq \mathbb{R}^{k}$ and $d \gg 0$, the dimension of $S_{d}^{r}(\Delta)$ is given by a polynomial of degree $k$, and a corollary of Theorem 2.12 is that a suitable analog of the Schumaker formula gives the top three coefficients of the polynomial, for any $k$.

\section{GRAdED ALGEBRA}

From our earlier discussion, it follows that we can think of a spline as a vector with polynomial entries, one polynomial for each maximal face, satisfying certain conditions ensuring that the resulting function (a priori only defined on individual faces) is globally a $C^{r}$ smooth function. Furthermore, we can add splines of the same order of smoothness. Given a $C^{r}$ spline represented as a vector of polynomials $\left(f_{1}, \ldots, f_{n}\right)$, multiplying the vector by a fixed polynomial $f$ gives $\left(f \cdot f_{1}, \ldots, f \cdot f_{n}\right)$, which is again a $C^{r}$ spline. This means that the set of splines is more than just a vector space; it is a module over the polynomial ring. In this section we build on this extra structure. Roughly speaking, the main advantage to this approach is that it packages all the vector spaces $S_{d}^{r}(\Delta)$ into a single object, where tools of algebra and algebraic geometry can be brought to bear on the problem. 
3.1. Rings and modules, Hilbert polynomial and series. Let $R$ denote the polynomial ring $\mathbb{R}\left[x_{1}, \ldots, x_{k+1}\right]$, in the setting of splines $k$ is the dimension of the ambient space. The polynomial ring has a special structure not shared by arbitrary rings: it has a grading by $\mathbb{Z}$ :

$$
R=\bigoplus_{i \in \mathbb{Z}} R_{i},
$$

where $R_{i}$ denotes the set of homogeneous (each monomial is of the same degree) polynomials of degree $i$, and if $r_{i} \in R_{i}$ and $r_{j} \in R_{j}$, then $r_{i} \cdot r_{j} \in R_{i+j}$. A graded $R$-module $M$ is defined in similar fashion. Of special interest is the case where $R_{0}$ is a field, for then each $M_{i}$ is a vector space. An $R$-module $M$ is free if it is isomorphic to $R^{m}$ for some $m \in \mathbb{N}$; a free rank one $R$-module with generator in degree $i$ is denoted $R(-i)$, so $R(i)_{j}=R_{i+j}$.

Example 3.1. Let $R=\mathbb{R}[x, y]$. The table below gives a bases for $R_{i}$ and $R(-2)_{i}$

\begin{tabular}{|c|c|c|}
\hline$i$ & $R_{i}$ & $R(-2)_{i}$ \\
\hline 0 & 1 & 0 \\
\hline 1 & $x, y$ & 0 \\
\hline 2 & $(x, y)^{2}$ & 1 \\
\hline 3 & $(x, y)^{3}$ & $x, y$ \\
\hline 4 & $(x, y)^{4}$ & $(x, y)^{2}$ \\
\hline$\vdots$ & $\vdots$ & $\vdots$ \\
\hline
\end{tabular}

$\diamond$

Definition 3.2. The Hilbert function $\operatorname{HF}(M, d)=\operatorname{dim}_{\mathbb{R}} M_{d}$.

Definition 3.3. The Hilbert series $H S(M, t)=\sum_{\mathbb{Z}} \operatorname{dim}_{\mathbb{R}} M_{i} t^{i}$.

Induction shows that $H S(R(-i), t)=t^{i} /(1-t)^{\ell}$ and $H F(R(-i), d)=\left(\begin{array}{c}d+k-i \\ d\end{array}\right)$ if $d \geq i$. For a finitely generated graded $R$-module $M$, the Hilbert function becomes polynomial for $d \gg 0$ ([34], Theorem 2.3.3), and is denoted $H P(M, d)$.

Example 3.4. For an example of a non-free $R$-module, let $R=\mathbb{R}[x, y]$, and $M=$ $R /\left\langle x^{2}, x y\right\rangle$. The table below gives a bases for $M_{i}$ and $M(-2)_{i}$

\begin{tabular}{|c|c|c|}
\hline$i$ & $M_{i}$ & $M(-2)_{i}$ \\
\hline 0 & 1 & 0 \\
\hline 1 & $x, y$ & 0 \\
\hline 2 & $y^{2}$ & 1 \\
\hline 3 & $y^{3}$ & $x, y$ \\
\hline 4 & $y^{4}$ & $y^{2}$ \\
\hline$\vdots$ & $\vdots$ & $\vdots$ \\
\hline
\end{tabular}

The respective Hilbert series are

$$
\begin{gathered}
H S(M, i)=\frac{1-2 t^{2}+t^{3}}{(1-t)^{2}} \text { and } \\
H S(M(-2), i)=\frac{t^{2}\left(1-2 t^{2}+t^{3}\right)}{(1-t)^{2}}
\end{gathered}
$$


3.2. Free resolutions. It is easy to compute the Hilbert series and Hilbert polynomial of a graded module from a finite free resolution.

Definition 3.5. A finite free resolution for an $R$-module $M$ is an exact sequence

$$
\mathbb{F}: 0 \rightarrow F_{k+1} \rightarrow \cdots \rightarrow F_{i} \stackrel{d_{i}}{\rightarrow} F_{i-1} \rightarrow \cdots \rightarrow F_{0} \rightarrow M \rightarrow 0,
$$

where the $F_{i}$ are free $R$-modules; $\mathbb{F}$ exists by the Hilbert syzygy theorem [19].

Example 3.6. For $R /\left\langle x^{2}, x y\right\rangle$, a finite free resolution is

$$
\begin{aligned}
0 \longrightarrow R(-3) \stackrel{\left[\begin{array}{c}
y \\
-x
\end{array}\right]}{\longrightarrow} R(-2)^{2} \stackrel{\left[\begin{array}{ll}
x^{2} & x y
\end{array}\right]}{\longrightarrow} R \longrightarrow R / I \longrightarrow 0 . \\
\text { The map }\left[x^{2}, x y\right] \text { sends } \begin{array}{c}
e_{1} \mapsto x^{2} \\
e_{2} \mapsto x y .
\end{array} \text { 长 }
\end{aligned}
$$

In order to have a map of graded modules, the basis elements of the source must have degree two, explaining the shifts in the free resolution. Here is where graded objects are useful: looking at a single fixed degree, we obtain an exact sequence of vector spaces; by Equation 2 the alternating sum of these dimensions is the dimension of $M_{d}$. For example, the alternating sum of the Hilbert series of the free modules above gives

$$
H S(M, i)=\frac{t^{3}-2 t^{2}+1}{(1-t)^{2}}
$$

which agrees with our earlier computation. $\diamond$

3.3. Grading in the spline setting. Billera and Rose observed in [9] that if $\hat{\Delta}$ is the simplicial complex obtained by embedding $\Delta$ in the plane $\left\{z_{k+1}=1\right\} \subseteq \mathbb{R}^{k+1}$ and forming the cone with the origin, then the set of splines (of all degrees) on $\hat{\Delta}$ is a graded module $S^{r}(\hat{\Delta})$ over $\mathbb{R}\left[x_{1}, \ldots, x_{k+1}\right]$, and $S^{r}(\hat{\Delta})_{d} \simeq S_{d}^{r}(\Delta)$.

In algebraic terms, we want the Hilbert series of $S^{r}(\hat{\Delta})$; if only asymptotic information (the dimension for $d \gg 0$ ) is needed, then it suffices to compute the Hilbert polynomial of $S^{r}(\hat{\Delta})$.

Lemma 3.7. [Billera-Rose, [9]] Let $P \subseteq \mathbb{R}^{k}$ be a $k$-dimensional polyhedral complex. Then there is a graded exact sequence:

$$
\begin{gathered}
0 \longrightarrow S^{r}(\hat{P}) \longrightarrow R^{f_{k}} \oplus R^{f_{k-1}^{0}}(-r-1) \stackrel{\phi}{\longrightarrow} R^{f_{k-1}^{0}} \longrightarrow N \longrightarrow 0 \\
\text { where } \phi=\left[\partial_{k} \mid \begin{array}{cccc}
l_{\tau_{1}}^{r+1} & & \\
& & \ddots & \\
& & l_{\tau_{m}}^{r+1}
\end{array}\right]
\end{gathered}
$$

Write $\left[\partial_{k} \mid D\right]$ for $\phi$. To describe $\partial_{k}$, note that the rows of $\partial_{k}$ are indexed by $\tau \in P_{k-1}^{0}$. If $\sigma_{1}, \sigma_{2}$ denote the $k$-faces adjacent to $\tau$, then in the row corresponding to $\tau$ the smoothness condition means that the only nonzero entries occur in the columns corresponding to $\sigma_{1}, \sigma_{2}$, and are $\pm(+1,-1)$. When $P$ is simplicial, $\partial_{k}$ is the top boundary map in the (relative) chain complex. Billera-Rose show $N$ is supported on primes of codimension at least two, which allows them to determine the top two coefficients of the Hilbert polynomial for arbitrary $k$; a refinement of this appears in Alfeld [3]. Both leave open the question of the $O\left(d^{k-2}\right)$ terms of $H P\left(S^{r}(\hat{\Delta}), d\right)$, which are determined in [33] and [35]. 


\section{LocAlizATion}

A key concept in many areas of mathematics is that of a quotient of an object $G$ by some subset $H$; the quotient $G / H$ is typically a simpler object. An analogous but less familiar operation is localization; rather than zeroing out $H$ as in the quotient construction, localization makes elements in the subset invertible.

\subsection{Basics of the construction.}

Definition 4.1. Let $R$ be a ring and $S$ a multiplicatively closed subset of $R$ containing 1. Define an equivalence relation on $\left\{\frac{r}{s} \mid r \in R, s \in S\right\}$ via

$$
\frac{r_{1}}{s_{1}} \sim \frac{r_{2}}{s_{2}} \text { if }\left(r_{1} s_{2}-r_{2} s_{1}\right) s_{3}=0 \text { for some } s_{3} \in S
$$

The localization $R_{S}$ is the set of equivalence classes; it is easily checked to be a ring.

The most common usages are when $S$ is either the set of all multiples of some element $\left\{1, r, r^{2}, \cdots\right\}$, or when $S$ is the complement of a prime ideal $\mathfrak{p}$. Recall that an ideal $\mathfrak{p}$ is prime if $a b \in \mathfrak{p}$ implies either $a \in \mathfrak{p}$ or $b \in \mathfrak{p}$; this condition means the complement is indeed multiplicatively closed. In particular, the process of localizing at a prime ideal $\mathfrak{p}$ makes every element outside $\mathfrak{p}$ invertible.

Example 4.2. In $\mathbb{Z},\langle 0\rangle$ is prime, so $S=\mathbb{Z} \backslash\{0\}$, and $\mathbb{Z}$ localized at $\langle 0\rangle$ is $\mathbb{Q}$. $\diamond$

Given a module $M$ over a ring $R$ and prime ideal $\mathfrak{p} \subseteq R$, then the localization $M_{\mathfrak{p}}$ is constructed as above; $M_{\mathfrak{p}}$ is an $R_{\mathfrak{p}}$-module, and is isomorphic to $M \otimes_{R} R_{\mathfrak{p}}$.

Example 4.3. Let $R=\mathbb{R}[x, y, z]$ and $M=R /\langle x y, x z\rangle$. Localizing $M$ and $R$ at the prime $\mathfrak{p}=\langle x\rangle$, we see that

$$
M_{\mathfrak{p}} \simeq R_{\mathfrak{p}} /\langle x\rangle R_{\mathfrak{p}},
$$

and localizing at the prime $\mathfrak{p}^{\prime}=\langle y, z\rangle$ we find that $M_{\mathfrak{p}^{\prime}} \simeq R_{\mathfrak{p}^{\prime}} /\langle y, z\rangle R_{\mathfrak{p}^{\prime}}$. So localization really does allow us to focus in on local properties. $\diamond$

Definition 4.4. A prime ideal $\mathfrak{p}$ is associated to a graded $R$-module $M$ if $\mathfrak{p}$ is the annihilator of some $m \in M$. Ass $(M)$ denotes the set of associated primes of $M$.

The reason that the associated primes of a module $M$ are important is that they are exactly the prime ideals at which the localization $M_{\mathfrak{p}} \neq 0$. A key fact we will need ([34], Theorem 6.1.3) is that localization preserves exact sequences.

4.2. Application: polyhedral splines. In [42], Schumaker obtained upper and lower bounds for splines on a planar polyhedral complex $P$. We sketch results of [27] yielding an analog of Theorem 1.1 in the polyhedral setting. The top two coefficients of the Hilbert polynomial depend on $f_{2}(P), f_{1}^{0}(P)$ and $r$, and agree with the Schumaker formula for the simplicial case. However, the constant term differs, and in a very interesting fashion. In addition to Schumaker's work on bounds, in [47], Yuzvinsky obtains results on freeness in the polyhedral setting.

Example 4.5. Let $P$ be the polygonal complex depicted below, and $P^{\prime}$ be a complex obtained by perturbing (any) vertex so that the affine spans of the three edges which connect boundary vertices to interior vertices are not concurrent. The following table gives the dimensions for $S_{d}^{r}(P)$ and $S_{d}^{r}\left(P^{\prime}\right)$ for small values of $r$, as long as $d \gg 0$. 


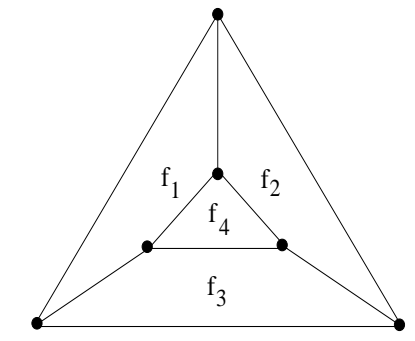

\begin{tabular}{|c|c|c|}
\hline$r$ & $\operatorname{dim}_{\mathbb{R}} S_{d}^{r}(P)$ & $\operatorname{dim}_{\mathbb{R}} S_{d}^{r}\left(P^{\prime}\right)$ \\
\hline 0 & $2 d^{2}+2$ & $2 d^{2}+1$ \\
\hline 1 & $2 d^{2}-6 d+10$ & $2 d^{2}-6 d+7$ \\
\hline 2 & $2 d^{2}-12 d+32$ & $2 d^{2}-12 d+25$ \\
\hline 3 & $2 d^{2}-18 d+64$ & $2 d^{2}-18 d+52$ \\
\hline 4 & $2 d^{2}-24 d+110$ & $2 d^{2}-24 d+91$ \\
\hline
\end{tabular}

Theorem 4.17 gives a complete explanation of this table. $\diamond$

We sketch the strategy: it follows from additivity of the Hilbert polynomial on exact sequences and Lemma 3.7 that obtaining the coefficient of $d^{k-2}$ in the Hilbert polynomial of $S^{r}(\hat{\Delta})$ is equivalent to obtaining the coefficient of $d^{k-2}$ in the Hilbert polynomial of $N$. Since

$$
N \simeq\left(\bigoplus_{\tau \in P_{k-1}^{0}} R / l_{\tau}^{r+1}\right) / \operatorname{im}\left(\partial_{k}\right)
$$

every element of $N$ is annihilated by some $r \in R$. Using localization, we first show that the codimension two associated primes of $N$ must be linear, then give a precise description of which codimension two linear primes actually occur. This leads to an explicit description of the submodule of $N$ supported in codimension two. Elements of this submodule are the only elements of $N$ which contribute to the $d^{k-2}$ coefficient of the Hilbert polynomial, and the formula follows.

Example 4.6. In Example 4.5, label the boundary vertices as 1,2,3, starting from the top vertex and moving clockwise, and the interior vertices 4,5,6 in the same way. Choose as an oriented basis for $\Delta_{1}^{0}$

$$
\{[41],[52],[63],[45],[56],[64]\}
$$

and for $\Delta_{2}$

$$
\{[1364],[1452],[2563],[465]\}
$$

Then the matrix for $\partial_{2}$ is

$$
\left[\begin{array}{cccc}
1 & -1 & 0 & 0 \\
0 & 1 & -1 & 0 \\
-1 & 0 & 1 & 0 \\
0 & 1 & 0 & -1 \\
0 & 0 & 1 & -1 \\
1 & 0 & 0 & -1
\end{array}\right]
$$


and so the matrix $\phi$ of Equation 6 is

$$
\phi=\left[\begin{array}{cccccccccc}
1 & -1 & 0 & 0 & l_{1}^{r+1} & 0 & 0 & 0 & 0 & 0 \\
0 & 1 & -1 & 0 & 0 & l_{2}^{r+1} & 0 & 0 & 0 & 0 \\
-1 & 0 & 1 & 0 & 0 & 0 & l_{3}^{r+1} & 0 & 0 & 0 \\
0 & 1 & 0 & -1 & 0 & 0 & 0 & l_{4}^{r+1} & 0 & 0 \\
0 & 0 & 1 & -1 & 0 & 0 & 0 & 0 & l_{5}^{r+1} & 0 \\
1 & 0 & 0 & -1 & 0 & 0 & 0 & 0 & 0 & l_{6}^{r+1}
\end{array}\right] .
$$

Let $p$ be the point where the affine spans of edges [14], [25], [36] meet, and let $\mathfrak{p}$ be the ideal of polynomials vanishing at $p$. So $\left\{l_{1}, l_{2}, l_{3}\right\} \subseteq \mathfrak{p}$ and $l_{4}, l_{5}, l_{6}$ are not in $\mathfrak{p}$. This means in the localization at $\mathfrak{p}$, the forms $l_{4}, l_{5}, l_{6}$ are units. Since $N_{\mathfrak{p}}$ is the cokernel of $\phi_{\mathfrak{p}}$, because $l_{4}, l_{5}, l_{6}$ are units, the images in $N_{\mathfrak{p}}$ of the last three rows are zero. In particular, $N_{\mathfrak{p}}$ is the cokernel of

$$
\phi_{\mathfrak{p}}=\left[\begin{array}{cccccccccc}
1 & -1 & 0 & 0 & l_{1}^{r+1} & 0 & 0 & 0 & 0 & 0 \\
0 & 1 & -1 & 0 & 0 & l_{2}^{r+1} & 0 & 0 & 0 & 0 \\
-1 & 0 & 1 & 0 & 0 & 0 & l_{3}^{r+1} & 0 & 0 & 0
\end{array}\right],
$$

$N_{\mathfrak{p}}$ has three generators, but quotienting by the first three columns of $\phi_{\mathfrak{p}}$ makes the three generators equal in the cokernel; in particular

$$
N_{\mathfrak{p}} \simeq R_{\mathfrak{p}} /\left\langle l_{1}^{r+1}, l_{2}^{r+1}, l_{3}^{r+1}\right\rangle
$$

Note that if we perturb a vertex so that the three lines are not concurrent, then one of $l_{1}, l_{2}, l_{3}$ will become a unit in $R_{\mathfrak{p}}$. This forces $N_{\mathfrak{p}}$ to vanish, and explains the difference between $S_{d}^{r}(P)$ and $S_{d}^{r}\left(P^{\prime}\right)$. $\diamond$

Lemma 4.7. Any prime ideal $\mathfrak{p}$ associated to $N$ contains a linear form $l_{\tau}$, for some $\tau \in P_{k-1}^{0}$.

Proof. From the description

$$
N \simeq\left(\bigoplus_{\tau \in P_{k-1}^{0}} R / l_{\tau}^{r+1}\right) / \operatorname{im}\left(\partial_{k}\right)
$$

it follows that if no $l_{\tau}$ is in $\mathfrak{p}$, then all the $l_{\tau}$ are invertible in $R_{\mathfrak{p}}$, so that $N_{\mathfrak{p}}$ vanishes.

Lemma 4.8. Let $\xi=V(\mathfrak{p})$ be a linear space. If $\sigma \in P_{k}$ has at most one facet whose linear span contains $\xi$, then every generator of $N$ corresponding to a facet of $\sigma$ is mapped to zero in the localization $N_{\mathfrak{p}}$.

Proof. In $R_{\mathfrak{p}}$, any $l_{\tau}$ with $\xi \nsubseteq V\left(l_{\tau}\right)$ becomes invertible. As $N$ is the cokernel of

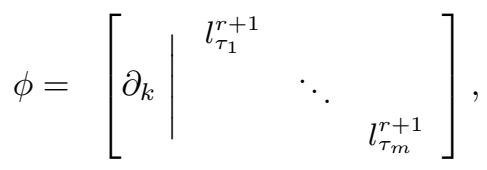

in the right hand diagonal submatrix $D$ of $\phi$, all the forms (or all save one) $l_{\tau}^{r+1}$ such that $\tau$ is a facet of $\sigma$ become units. As the column of the left hand $\left(\partial_{k}\right)$ matrix corresponding to $\sigma$ has nonzero entries only in rows corresponding to facets of $\sigma$, this means that every generator corresponding to a facet of $\sigma$ has zero image in the localization, and the result follows.

Theorem 4.9. Any codimension two prime ideal $\mathfrak{p}$ associated to $N$ is of the form $\left\langle l_{\tau_{1}}, l_{\tau_{2}}\right\rangle$ for $\tau_{i} \in P_{d-1}^{0}$ such that $V\left(l_{\tau_{1}}, l_{\tau_{2}}\right)$ has codimension two. 
Proof. If there do not exist two $l_{i}$ as above, then by Lemma 4.7, $V(\mathfrak{p})$ is contained in exactly one hyperplane which is the linear span of $\tau \in P_{k-1}^{0}$. Thus, in $R_{\mathfrak{p}}$, all but one of the $l_{\tau}$ become units, and the proof of Lemma 4.8 shows that $N_{\mathfrak{p}}$ vanishes.

Theorem 4.9 gives an explicit set of candidates for the codimension two primes of $N$. By Billera and Rose [9] all associated primes of $N$ have codimension at least two (this also follows from the argument above), so the theorem identifies all candidates for the associated primes of minimal codimension. In order to determine exactly which codimension two linear primes are actually associated to $N$, we introduce a graph, which depends on both combinatorics and geometry of $P$. In the simplicial case, the codimension two associated primes are exactly the vertices of $P$. In the polyhedral case, the geometry is more subtle.

Definition 4.10. Let $P$ be a $k$-dimensional polyhedral complex embedded in $\mathbb{R}^{k}$, and $\xi$ a codimension two linear subspace. $G_{\xi}(P)$ is a graph whose vertices correspond to those $\sigma \in P_{k}$ such that there exists a $(k-1)$-face of $\sigma$ whose linear span contains $\xi$. Two vertices of $G_{\xi}(P)$ are joined iff the corresponding $k$-faces share a common $(k-1)$-face whose linear span contains $\xi$.

Example 4.11. In Example 4.5, each interior vertex $v$ of $P$ has $G_{v}(P)$ a triangle. Additionally, if $\xi$ is the point at which affine spans of the three edges connecting interior vertices to boundary vertices meet, then $G_{\xi}(P)$ is as below, where $v_{i}$ corresponds to the facet labeled $f_{i}$ in the figure of Example 4.5.

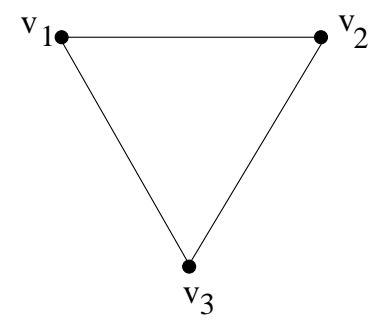

Let $P^{\prime}$ be obtained by moving the top vertex of $P$ a bit to the right. Then $P$ and $P^{\prime}$ are combinatorially equivalent, but in $P^{\prime}$ there are no sets of $\geq 3$ concurrent $V\left(l_{\tau}\right), \tau \in P_{d-1}^{0}$, except at the interior vertices. In particular, $G_{\xi}\left(P^{\prime}\right)$ is acyclic. $\diamond$

Lemma 4.12. For any $\sigma \in P_{k}$, there are at most two facets of $\sigma$ whose linear spans contain a given codimension two linear space $\xi$.

Proof. Suppose the linear spans of three facets $\tau_{1}, \tau_{2}, \tau_{3}$ of $\sigma$ meet in a codimension two linear space $\xi$. For each $V\left(l_{\tau_{i}}\right), \sigma$ lies on one side of the hyperplane; so $\sigma$ lies between $V\left(l_{\tau_{1}}\right)$ and $V\left(l_{\tau_{2}}\right)$. Since $V\left(l_{\tau_{3}}\right)$ contains

$$
\xi=V\left(l_{\tau_{1}}\right) \cap V\left(l_{\tau_{2}}\right),
$$

this means $V\left(l_{\tau_{3}}\right)$ would split $\sigma$, a contradiction.

Corollary 4.13. $G_{\xi}(P)$ is homotopic to a disjoint union of circles and segments.

Proof. By Lemma 4.12, the valence of any vertex $v \in G_{\xi}(P)$ is at most two. 
Theorem 4.14. For a polyhedral $P$ and codimension two linear space $\xi=V(\mathfrak{p})$,

$$
N_{\mathfrak{p}} \simeq \bigoplus_{\psi \in H_{1}\left(G_{\xi}(P)\right)}\left(R / I_{\psi}\right)_{\mathfrak{p}}
$$

where $\psi \in H_{1}\left(G_{\xi}(P)\right)$ means $\psi$ is a component of $G_{\xi}(P)$ homotopic to $S^{1}$, and

$$
\left.I_{\psi}=\left\langle l_{\tau}^{r+1}\right| \tau \in P_{k-1}^{0} \text { corresponds to an edge of } \psi\right\rangle .
$$

Proof. By Corollary 4.13, $G_{\xi}(P)$ consists of a disjoint union of cycles and segments. By Lemma 4.8, all generators of $N$ which lie in a segment are mapped to zero in the localization $N_{\mathfrak{p}}$. For each $k$-face $\sigma$ corresponding to a vertex in a cycle, note that there are two $(k-1)$-faces $\tau_{1}, \tau_{2}$ of $\sigma$ such that $l_{\tau_{1}}, l_{\tau_{2}}$ are not units in $R_{\mathfrak{p}}$; every other linear form defining a facet of $\sigma$ becomes a unit. Reducing the column of $\partial_{k}$ corresponding to $\sigma$ by the columns of $D_{\mathfrak{p}}$ having a unit entry gives a column with nonzero entries only in rows corresponding to $\tau_{1}$ and $\tau_{2}$. Repeating the process shows that the cycle corresponds to a principal submodule of $N_{\mathfrak{p}}$, with the generator quotiented by the $(r+1)^{s t}$ powers of the forms corresponding to the edges of the cycle.

Proposition 4.15. Let $\mathcal{P}$ be the set of all codimension two associated primes of $N$. Then there is an exact sequence

$$
0 \longrightarrow K \longrightarrow N \longrightarrow \bigoplus_{\substack{\psi \in H_{1}\left(G_{V}(Q)\right) \\ Q \in \mathcal{P}}} R / I_{\psi} \longrightarrow C \longrightarrow 0
$$

where $K$ and $C$ are supported in codimension at least three.

Proof. The reasoning in the proof of Theorem 4.14 shows that if $\xi=V(Q)$ with $Q \in \mathcal{P}$, then

$$
\bigoplus_{\psi \in H_{1}\left(G_{V(Q)}(P)\right)} R / I_{\psi}
$$

is exactly the cokernel of the submatrix of $\left[\partial_{k} \mid D\right]$ obtained by deleting those rows indexed by $\tau \in P_{k-1}^{0}$ such that $\xi \notin \operatorname{conv}(\tau)$. An application of the snake lemma ([34], Lemma 8.1.1) then shows that

$$
N \longrightarrow \bigoplus_{\psi \in H_{1}\left(G_{\xi}(P)\right)} R / I_{\psi} \longrightarrow 0
$$

is exact. Taking the sum of such maps over all $Q \in \mathcal{P}$ yields the exact sequence of the proposition. Theorem 4.14 shows that upon localizing this sequence at any prime $Q \in \mathcal{P}$, the localizations $C_{Q}$ and $K_{Q}$ vanish, hence $K$ and $C$ are supported in codimension at least three.

Lemma 4.16. [Schumaker, [41]] Let $I_{\psi}=\left\langle l_{1}^{r+1}, \ldots, l_{n}^{r+1}\right\rangle \subseteq \mathbb{R}\left[x_{1}, \ldots, x_{k+1}\right]$ be a codimension two ideal, minimally generated by the $n$ given elements. Define

$$
\begin{array}{ccc}
\alpha(\psi) & = & \left\lfloor\frac{r+1}{n-1}\right\rfloor, \\
s_{1}(\psi) & = & (n-1) \alpha(\psi)+n-r-2, \\
s_{2}(\psi) & = & r+1-(n-1) \alpha(\psi) .
\end{array}
$$

Then the minimal free resolution of $R / I_{\psi}$ is:

$$
0 \longrightarrow \begin{aligned}
& R(-r-1-\alpha(\psi))^{s_{1}(\psi)} \\
& R(-r-2-\alpha(\psi))^{s_{2}(\psi)}
\end{aligned} \longrightarrow R(-r-1)^{n} \longrightarrow R \longrightarrow R / I_{\psi} \longrightarrow 0 .
$$


Proof. See Theorem 3.1 of [40]; the key step involves showing that a certain matrix has full rank, which was established by Schumaker in [42].

It follows from Lemma 4.16 that the Hilbert polynomial of $R / I_{\psi}$ is given by:

$$
\left(\begin{array}{c}
k+d \\
k
\end{array}\right)-n\left(\begin{array}{c}
k+d-r-1 \\
k
\end{array}\right)+s_{1}(\psi)\left(\begin{array}{c}
k+d-r-1-\alpha(\psi) \\
k
\end{array}\right)+s_{2}(\psi)\left(\begin{array}{c}
k+d-r-2-\alpha(\psi) \\
k
\end{array}\right) .
$$

Theorem 4.17. If $P$ is a hereditary planar polyhedral complex, then

$$
H P\left(S^{r}(\hat{\Delta}), d\right)=\frac{f_{2}}{2} d^{2}+\frac{3 f_{2}-2(r+1) f_{1}^{0}}{2} d+f_{2}+\left(\left(\begin{array}{l}
r \\
2
\end{array}\right)-1\right) f_{1}^{0}+\sum_{\psi_{j} \in H_{1}\left(G_{\xi_{i}}(P)\right)} c_{j},
$$

where

$$
\begin{aligned}
c_{j}=1 & -n\left(\psi_{j}\right)\left(\begin{array}{l}
r \\
2
\end{array}\right)+s_{1}\left(\psi_{j}\right)\left(\begin{array}{c}
r+\alpha\left(\psi_{j}\right) \\
2
\end{array}\right)+s_{2}\left(\psi_{j}\right)\left(\begin{array}{c}
r+\alpha\left(\psi_{j}\right)+1 \\
2
\end{array}\right) \\
= & \left(\begin{array}{c}
r+2 \\
2
\end{array}\right)+\frac{\alpha\left(\psi_{j}\right)}{2}\left(2 r+3+\alpha\left(\psi_{j}\right)-n\left(1+\alpha\left(\psi_{j}\right)\right)\right) .
\end{aligned}
$$

We close by applying Theorem 4.17 to Example 4.5. As we saw in Example 4.11, there are four $\xi$ at which $H_{1}\left(G_{\xi}(P)\right) \neq 0$, and each $I_{\psi}$ has three generators. Hence the $c_{j}$ are all the same, and equal to

$$
\left(\begin{array}{c}
r+2 \\
2
\end{array}\right)+\frac{\alpha\left(\psi_{j}\right)}{2}\left(2 r+3+\alpha\left(\psi_{j}\right)-3\left(1+\alpha\left(\psi_{j}\right)\right)\right) .
$$

which simplifies to

$$
\left(\begin{array}{c}
r+2 \\
2
\end{array}\right)+\left\lfloor\frac{r+1}{2}\right\rfloor\left(r-\left\lfloor\frac{r+1}{2}\right\rfloor\right) .
$$

Theorem 4.17 yields:

\begin{tabular}{|c|c|c|c|c|}
\hline$r$ & $\operatorname{dim}_{\mathbb{R}} S_{d}^{r}(P)$ & $\frac{f_{2}}{2} d^{2}+\frac{3 f_{2}-2(r+1) f_{1}^{0}}{2} d$ & $\left.f_{2}+\left(\begin{array}{c}r \\
2\end{array}\right)-1\right) f_{1}^{0}$ & $4\left(\left(\begin{array}{c}r+2 \\
2\end{array}\right)+\alpha(r-\alpha)\right)$ \\
\hline 0 & $2 d^{2}+2$ & $2 d^{2}$ & -2 & 4 \\
\hline 1 & $2 d^{2}-6 d+10$ & $2 d^{2}-6 d$ & -2 & 12 \\
\hline 2 & $2 d^{2}-12 d+32$ & $2 d^{2}-12 d$ & 4 & 28 \\
\hline 3 & $2 d^{2}-18 d+64$ & $2 d^{2}-18 d$ & 16 & 48 \\
\hline 4 & $2 d^{2}-24 d+110$ & $2 d^{2}-24 d$ & 34 & 76 \\
\hline
\end{tabular}

For the configuration $P^{\prime}$ obtained by perturbing a vertex in Example 3.4 so the three edges defining $\xi$ no longer meet, there are only three nontrivial $c_{j}$, hence

$$
\operatorname{dim}_{\mathbb{R}} S_{d}^{r}\left(P^{\prime}\right)=\operatorname{dim}_{\mathbb{R}} S_{d}^{r}(P)-\left(\begin{array}{c}
r+2 \\
2
\end{array}\right)-\alpha(r-\alpha)
$$


4.3. Application: vanishing of homology. As a second application of localization, we prove Theorem 2.12. Two key properties of localization [19] are that it preserves exactness, and it commutes with homology. Write $1_{\alpha}$ for the unit of $\mathcal{R} / \mathcal{J}(\alpha)$. We have the complex:

$$
\cdots \longrightarrow \bigoplus_{\alpha \in \Delta_{i+1}^{0}} \mathcal{R} / \mathcal{J}(\alpha) \stackrel{\partial_{i+1}}{\longrightarrow} \bigoplus_{\beta \in \Delta_{i}^{0}} \mathcal{R} / \mathcal{J}(\beta) \stackrel{\partial_{i}}{\longrightarrow} \bigoplus_{\gamma \in \Delta_{i-1}^{0}} \mathcal{R} / \mathcal{J}(\gamma) \stackrel{\partial_{i-1}}{\longrightarrow} \cdots
$$

Let $\mathfrak{p}$ be a prime ideal such that $\mathcal{J}(\gamma) \nsubseteq \mathfrak{p}$, for any $\gamma \in \Delta_{i-1}^{0}$. Then

$$
\begin{gathered}
\bigoplus_{\gamma \in \Delta_{i-1}^{0}} \mathcal{R} / \mathcal{J}(\gamma)_{\mathfrak{p}}=0, \text { so } \\
H_{i}(\mathcal{R} / \mathcal{J})_{\mathfrak{p}}=\bigoplus_{\beta \in \Delta_{i}^{0}} \mathcal{R} / \mathcal{J}(\beta)_{\mathfrak{p}} /\left(\operatorname{im}\left(\partial_{i+1}\right)\right)_{\mathfrak{p}}
\end{gathered}
$$

Now, if $\mathcal{J}(\beta) \nsubseteq \mathfrak{p}$ for any $\beta \in \Delta_{i}^{0}$, then

$$
\begin{gathered}
\bigoplus_{\beta \in \Delta_{i}^{0}} \mathcal{R} / \mathcal{J}(\beta)_{\mathfrak{p}}=0, \text { so } \\
H_{i}(\mathcal{R} / \mathcal{J})_{\mathfrak{p}}=0,
\end{gathered}
$$

and we're done. So suppose $\mathcal{J}(\beta) \subseteq \mathfrak{p}$, for some (possibly several) $\beta \in \Delta_{i}^{0}$. For $\alpha \in \Delta_{i+1}^{0}$, the map $\partial_{i+1}$ takes $1_{\alpha}$ to a signed sum of $1_{\beta}$, where $\beta$ is a facet of $\alpha$. Localization at $\mathfrak{p}$ sends $1_{\beta}$ to zero if $\mathcal{J}(\beta) \nsubseteq \mathbb{p}$. Because $\Delta$ is simplicial, two facets of $\alpha$ intersect in a face of dimension $i-1$, and the assumption that $\mathfrak{p}$ does not contain $\mathcal{J}(\gamma)$ for any $i-1$ face $\gamma$ implies that in the localization of $\partial_{i+1}\left(1_{\alpha}\right)$, at most one $1_{\beta}$ is nonzero, and some $1_{\beta}$ is nonzero only if $\mathcal{J}(\beta) \subseteq \mathfrak{p}$. Thus $\partial_{i+1_{\mathfrak{p}}}\left(1_{\alpha}\right)=$ $1_{\beta}$ if $\beta \subseteq \alpha$ and $\mathcal{J}(\beta) \subseteq \mathfrak{p}$, so $\partial_{i+1_{\mathfrak{p}}}$ is surjective and $H_{i}(\mathcal{R} / \mathcal{J})_{\mathfrak{p}}=0$.

We have shown that $H_{i}(\mathcal{R} / \mathcal{J})_{\mathfrak{p}}=0$ if $\mathfrak{p} \nsupseteq \mathcal{J}(\gamma)$ for any $\gamma \in \Delta_{i-1}^{0}$. Since $\mathfrak{p}$ is prime, this means that if $H_{i}(\mathcal{R} / \mathcal{J})_{\mathfrak{p}} \neq 0$, then $\mathfrak{p} \supseteq I(\gamma)$, for some $\gamma \in \Delta_{i-1}^{0}$. If we can show that $H_{i}(\mathcal{R} / \mathcal{J})_{I(\gamma)}=0$ for all $\gamma \in \Delta_{i-1}^{0}$, then since $I(\gamma)$ is of codimension $k-i+1, H_{i}(\mathcal{R} / \mathcal{J})$ is supported on primes of codimension at least $k-i+2$, which will conclude the proof.

Suppose $\mathfrak{p}=I(\gamma)$, some $\gamma \in \Delta_{i-1}^{0}$. Then the localized complex splits into a direct sum of subcomplexes, of which two types can contribute to $H_{i}(\mathcal{R} / \mathcal{J})_{\mathfrak{p}}$. The first type are those of the following form, with one piece for each $i-1$ face $\gamma_{j}$ such that $\hat{\gamma}_{j} \subseteq V(I(\gamma))$ :

$$
\cdots \longrightarrow \bigoplus_{\substack{\gamma_{j} \in \alpha \\ \alpha \in \Delta_{i+1}}} \mathcal{R} / \mathcal{J}(\alpha)_{I(\gamma)} \longrightarrow \bigoplus_{\substack{\gamma_{j} \in \beta \\ \beta \in \Delta_{i}^{0}}} \mathcal{R} / \mathcal{J}(\beta)_{I(\gamma)} \longrightarrow \mathcal{R} / \mathcal{J}\left(\gamma_{j}\right)_{I(\gamma)} \longrightarrow 0
$$

The map $\partial_{i_{I(\gamma)}}$ sends each summand surjectively to $\mathcal{R} / \mathcal{J}\left(\gamma_{j}\right)_{I(\gamma)}$, so the kernel of $\partial_{i_{I(\gamma)}}$ is generated by pairs of units with opposite orientations (e.g. $1_{\beta_{i}}-1_{\beta_{j}}$ ), along with generators of the form $l_{\beta_{i}}^{r+1} \cdot 1_{\beta_{j}}$, where $l_{\beta_{i}}^{r+1} \in \mathcal{J}\left(\beta_{i}\right)$, but $l_{\beta_{i}}^{r+1} \notin \mathcal{J}\left(\beta_{j}\right)$. An $i+1$ face $\alpha$ in the above subcomplex has a pair of $i$ faces $\beta_{i}, \beta_{j}$ which intersect in $\gamma_{j}$ (again, we make use of the fact that $\Delta$ is simplicial), and clearly $\partial_{i+1_{I(\gamma)}}\left(1_{\alpha}\right)=$ $1_{\beta_{i}}-1_{\beta_{j}}$, which generate all elements of the kernel of the first type mentioned above. For generators of the second type, notice that modulo the image of $\partial_{\left.i+1_{I(\gamma)}\right)}$, $l_{\beta_{i}}^{r+1} \cdot 1_{\beta_{j}}=l_{\beta_{i}}^{r+1} \cdot 1_{\beta_{i}}$, so is zero in homology. Thus, these subcomplexes do not contribute to $H_{i}(\mathcal{R} / \mathcal{J})_{\mathfrak{p}}$. 
The second type of subcomplex which may contribute to $H_{i}(\mathcal{R} / \mathcal{J})_{\mathfrak{p}}$ are those with $V(I(\gamma)) \subseteq V\left(I\left(\beta_{d}\right)\right), \beta_{d} \in \Delta_{i}^{0}$, but where $\beta_{d}$ does not contain an $i-1$ face $\gamma_{d}$ such that $\hat{\gamma}_{d} \subseteq V(I(\gamma))$. These complexes take the form:

$$
\cdots \longrightarrow \bigoplus_{\substack{\beta_{d} \in \alpha \\ \alpha \in \Delta_{i+1}}} \mathcal{R} / \mathcal{J}(\alpha)_{I(\gamma)} \longrightarrow \mathcal{R} / \mathcal{J}\left(\beta_{d}\right)_{I(\gamma)} \longrightarrow 0
$$

It is easy to check that the localized $\partial_{i+1}$ map is surjective, and hence for these subcomplexes we also have $H_{i}(\mathcal{R} / \mathcal{J})_{\mathfrak{p}}=0$, which concludes the proof. Using the technical tool of spectral sequences, one can show more:

Theorem 4.18. [[33]] If $\Delta \subseteq \mathbb{R}^{k}$ is topologically trivial, then $S^{r}(\hat{\Delta})$ is a free module if and only if $H_{i}(\mathcal{R} / \mathcal{J})=0$ for all $i \leq d-1$.

Corollary 4.19. [[33]] If $S^{r}(\hat{\Delta})$ is free, then the Hilbert series for $S^{r}(\hat{\Delta})$ is determined entirely by local data $H S\left(S^{r}(\hat{\Delta}), t\right)=\sum_{i=0}^{k}(-1)^{k-i} H S\left(\mathcal{R} / \mathcal{J}_{i}, t\right)$.

Proof. Immediate from Theorem 4.18 and Equation 2.

Example 4.20. Consider the symmetric octahedron pictured below.

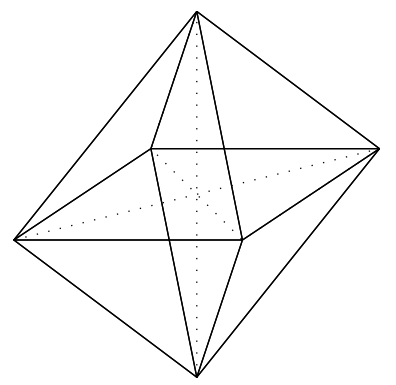

By directly analyzing the maps $\partial_{i}$ it is possible to show that $H_{2}(\mathcal{R} / \mathcal{J})=$ $H_{1}(\mathcal{R} / \mathcal{J})=0$, hence by Theorem $4.18, S^{r}(\hat{\Delta})$ is free for all $r$. By Corollary 4.19 , the Hilbert series is given as the alternating sum

$$
H S\left(S^{r}(\hat{\Delta}), t\right)=\sum_{i=0}^{3}(-1)^{3-i} H S\left(\mathcal{R} / \mathcal{J}_{i}, t\right)=\frac{1+3 t^{r+1}+3 t^{2 r+2}+t^{3 r+3}}{(1-t)^{4}}
$$

The computation above is simple because we may translate so that the $l_{\tau}$ are all coordinate hyperplanes-this example is very nongeneric. It is easy to identify the generators for $S^{r}(\hat{\Delta})$; for example, in degree $r+1$ there is a generator which is $z^{r+1}$ on the top four simplices, and zero on the bottom four; the other two generators of degree $r+1$ come from symmetry. Continue in this fashion. $\diamond$

\section{INVERSE SYSTEMS AND POWERS OF LINEAR FORMS}

A century ago, Macaulay [26] defined the notion of an inverse system.

Definition 5.1. Let $S=\mathbb{R}\left[y_{0}, \ldots, y_{n}\right]$. We think of $S$ both as a ring, isomorphic to $R$, and as an $R$-module where the action $R_{i} \times S_{j} \rightarrow S_{j-i}$ is that given by partial differentiation. For an ideal $I \subseteq R$, the inverse system $I^{-1}$ is the set of elements of $S$ which are annihilated by this action. 
5.1. Powers of linear forms and fatpoints. There is a beautiful connection between ideals generated by powers of linear forms occurring in the complexes $\mathcal{R} / \mathcal{J}$ of $\S 2$, and certain ideals defining zero dimensional objects in projective space.

Definition 5.2. Let $p_{i}=\left[\begin{array}{lllll}p_{i 0}: p_{i 1}: \cdots: p_{i n}\end{array}\right] \in \mathbb{P}^{n}, I\left(p_{i}\right)=P_{i} \subseteq R=$ $\mathbb{R}\left[x_{0}, \ldots, x_{n}\right]$. A fatpoints ideal is an ideal of the form $I=\cap_{i=1}^{m} \mathfrak{p}_{i}^{\alpha_{i}}, \alpha_{i} \geq 1$.

Theorem 5.3. [Ensalem and Iarrobino, [20]] For a fatpoints ideal $I=\mathfrak{p}_{1}^{n_{1}+1} \cap$ $\cdots \cap \mathfrak{p}_{s}^{n_{s}+1}$, let $l_{p_{i}}=\sum_{j=0}^{n} p_{i_{j}} y_{j}$. Then $I^{-1}=\operatorname{ann}_{S}(I)$ may be described as follows:

$$
\left(I^{-1}\right)_{j}= \begin{cases}S_{j} & \text { for } j \leq \max \left\{n_{i}\right\} \\ l_{p_{1}}^{j-n_{1}} S_{n_{1}}+\cdots+l_{p_{s}}^{j-n_{s}} S_{n_{s}} & \text { for } j \geq \max \left\{n_{i}+1\right\}\end{cases}
$$

and

$$
\operatorname{dim}_{\mathbb{R}}\left(I^{-1}\right)_{j}=\operatorname{dim}_{\mathbb{R}}(R / I, j)
$$

Holding $j-n_{i}=t_{i}$ fixed and letting $j$ and $n_{i}$ vary yields information about $\left\langle l_{1}^{t_{1}}, \ldots, l_{s}^{t_{s}}\right\rangle$, depending on an infinite family of ideals of fatpoints.

5.2. Application: planar splines of mixed smoothness. The paper [21] uses inverse systems to study splines of mixed smoothness. First, some preliminaries.

Corollary 5.4. Let $l_{1}, \ldots, l_{s}$ be s pairwise linearly independent homogeneous linear forms in $S=\mathbb{R}\left[y_{0}, y_{1}\right], 0<\alpha_{1} \leq \cdots \leq \alpha_{s}$ be integers, and let $J=\left\langle l_{1}^{\alpha_{1}}, \ldots, l_{s}^{\alpha_{s}}\right\rangle$. Then for each $t \in \mathbb{N}$, the vector space $J_{t}$ has the maximum dimension possible:

$$
\operatorname{dim}_{d} J_{t}=\min \left\{t+1, \sum_{i=1}^{s} \max \left\{t-\alpha_{i}+1,0\right\}\right\} .
$$

Proof. By Theorem 5.3, given an integer $t \geq 0$,

$$
\operatorname{dim}_{d} J_{t}=\operatorname{dim}_{d}(R / I, t)
$$

where

$$
I=\wp_{1}^{t-\alpha_{1}+1} \cap \ldots \cap \wp_{s}^{t-\alpha_{s}+1}
$$

and $\wp_{1}, \ldots, \wp_{s}$ are the ideals of the points corresponding to $l_{1}, \ldots, l_{s}$ (here we use the convention that $\mathfrak{p}^{r}=R$ if $\left.r \leq 0\right)$. Now $I$ is a principal ideal generated by a form $F$ of degree $d_{t}$, where

$$
d_{t}=\sum_{i=1}^{s} \max \left\{t-\alpha_{i}+1,0\right\} .
$$

So $\operatorname{dim}_{d} J_{t}=H(R / I, t)=\min \left(t+1, d_{t}\right)$.

Corollary 5.5. Let $0<\alpha_{1} \leq \alpha_{2} \cdots \leq \alpha_{t}$ and $J=\left\langle l_{1}^{\alpha_{1}}, \ldots, l_{t}^{\alpha_{t}}\right\rangle$. Then if $m \geq 2$ :

$$
l_{m+1}^{\alpha_{m+1}} \notin\left\langle l_{1}^{\alpha_{1}}, \ldots, l_{m}^{\alpha_{m}}\right\rangle \Leftrightarrow \alpha_{m+1} \leq \frac{\sum_{i=1}^{m} \alpha_{i}-m}{m-1} .
$$

Proof. Let $J_{m}=\left\langle l_{1}^{\alpha_{1}}, \ldots, l_{m}^{\alpha_{m}}\right\rangle$. Then $l_{m+1}^{\alpha_{m+1}} \notin J_{m}$ if and only if $\left(J_{m}\right)_{\alpha_{m+1}} \neq$ $\left(J_{m+1}\right)_{\alpha_{m+1}}$. By Corollary 5.4,

$$
\begin{aligned}
\operatorname{dim}\left(J_{m}\right)_{\alpha_{m+1}} & =\min \left\{\alpha_{m+1}+1, \sum_{i=1}^{m}\left(\alpha_{m+1}-\alpha_{i}+1\right)\right\} \\
\operatorname{dim}\left(J_{m+1}\right)_{\alpha_{m+1}} & =\min \left\{\alpha_{m+1}+1, \sum_{i=1}^{m+1}\left(\alpha_{m+1}-\alpha_{i}+1\right)\right\}
\end{aligned}
$$


Hence, $\left(J_{m}\right)_{\alpha_{m+1}} \neq\left(J_{m+1}\right)_{\alpha_{m+1}}$ if and only if

$$
\alpha_{m+1}+1>\sum_{i=1}^{m}\left(\alpha_{m+1}-\alpha_{i}+1\right)
$$

which simplifies to the above condition.

From now on, when we write $J=\left\langle l_{1}^{\alpha_{1}}, \ldots, l_{t}^{\alpha_{t}}\right\rangle$, we require the exponent vector $\alpha=\left(\alpha_{1}, \ldots, \alpha_{t}\right)$ of $J$ satisfies the conditions of Corollary 5.5, so that we have a minimal generating set for $J$ : for each integer $m \in 2 \ldots t-1, \alpha_{m+1} \leq \frac{\sum_{i=1}^{m} \alpha_{i}-m}{m-1}$. By Corollary 5.4, we also have the following:

Theorem 5.6. Let $J=\left\langle l_{1}^{\alpha_{1}}, \ldots, l_{t}^{\alpha_{t}}\right\rangle$ with exponent vector of $J$ as above and $d_{i}$ as in Corollary 5.4. Then

$$
H(S / J, i)=\max \left\{0, i+1-d_{i}\right\} .
$$

The least integer $\Omega$ for which $H(S / J, \Omega)=0$ is the least integer $p$ such that $p+1-d_{p} \leq 0$; equivalently $p<\sum_{i=1}^{t} \max \left\{p-\alpha_{i}+1,0\right\}$. Thus, $d_{\Omega-1} \leq \Omega-1$ and $\Omega<d_{\Omega}$; the socle degree of $S / J$ is $\Omega-1$. Since all the minimal generators of $J$ occur in degree at most one greater than the socle degree of $S / J$, we see that $\Omega \geq \alpha_{i}$ for all $i$.

$$
\Omega=\left\lfloor\frac{\sum_{i=1}^{t} \alpha_{i}-t}{t-1}\right\rfloor+1
$$

Theorem 5.7. [Geramita-Schenck [21]] Let $J$ be an ideal minimally generated by $\left\langle l_{1}^{\alpha_{1}}, \ldots, l_{t}^{\alpha_{t}}\right\rangle$, so that $\Omega-1$ is the socle degree of $S / J$. Then $J$ has resolution

$$
\begin{aligned}
& S(-\Omega-1)^{a} \\
& 0 \longrightarrow \underset{S(-\Omega)^{t-1-a}}{\oplus} \longrightarrow \oplus_{i=1}^{t} S\left(-\alpha_{i}\right) \longrightarrow J \longrightarrow 0 \text {, }
\end{aligned}
$$

where

$$
a=H(S / J, \Omega-1)=\sum_{i=1}^{t} \alpha_{i}+(1-t) \cdot \Omega .
$$

The proof uses the Hilbert Syzygy Theorem and the Hilbert-Burch theorem, which may be found in [19]. Theorem 5.7 generalizes Schumaker's result in Lemma 4.16 to allow varying smoothness. If $\alpha \in \mathbb{N}_{1}^{f_{1}^{0}}$ is an integer vector representing the order of smoothness across the interior edges, then this yields a simple formula for the Hilbert polynomial of $S_{d}^{\alpha}(\Delta)$ for planar splines of mixed smoothness on $\Delta$.

5.3. A conjecture in algebraic geometry. Suppose $\left\{p_{1}, \ldots, p_{n}\right\}$ is a collection of points in the plane, and $m_{i} \in \mathbb{N}$, with $I_{X}=\cap \mathfrak{p}_{i}^{m_{i}}$. A polynomial $f(x, y)$ vanishes with multiplicity $m_{i}$ at $p_{i}$ exactly when all partial derivatives of $f$ of order $\leq m_{i}-1$ vanish at $p_{i}$. This places $\left(\begin{array}{c}m_{i}-1 \\ 2\end{array}\right)$ independent constraints on $f$. If the $p_{i}$ are in general position, the natural hope is that the conditions from distinct points do not interact. Hence if we homogenize the problem, our expectation is that the Hilbert function of $R / I_{X}$ should be

$$
H F\left(R / I_{X}, j\right)=\left(\begin{array}{c}
j+2 \\
2
\end{array}\right)-\sum_{i=1}^{n}\left(\begin{array}{c}
m_{i}+1 \\
2
\end{array}\right)
$$

as soon as $j$ is sufficiently large. This naive hope is false: 
Example 5.8. Consider the space of conics through two double points. Then

$$
\left(\begin{array}{c}
2+2 \\
2
\end{array}\right)-2\left(\begin{array}{c}
2+1 \\
2
\end{array}\right)=6-6=0
$$

so there should be no such conics. But if $V(l)$ is the line connecting the two points, then $l^{2} \in \mathfrak{p}_{1}^{2} \cap \mathfrak{p}_{2}^{2}$. Similar behavior occurs for quartics through five double points:

$$
\left(\begin{array}{c}
4+2 \\
2
\end{array}\right)-5\left(\begin{array}{c}
2+1 \\
2
\end{array}\right)=15-15=0
$$

but the estimate fails: the space of conics has dimension six, and five points impose at most five conditions, so there is a conic $c \in \cap_{i=1}^{5} \mathfrak{p}_{i}$ and $c^{2} \in \cap_{i=1}^{5} \mathfrak{p}_{i}^{2}$. $\diamond$

A conjecture of Segre-Harbourne-Gimigliano-Hirschowitz is that this kind of behavior is the only pathology:

Conjecture 5.9. If a fatpoints ideal $I=\cap \mathfrak{p}_{i}^{m_{i}}$ supported at general points $p_{i}$ fails to have the expected Hilbert function $\operatorname{HF}(R / I, d)$, then the linear system $L=$ $d E_{0}-\sum m_{i} E_{i}$ on the blowup of $\mathbb{P}^{2}$ at the $p_{i}$ contains $a-1$ curve $E$ with $E \cdot L \leq-2$.

This statement is opaque, but a nice elementary exposition appears in Miranda's paper [28]. The takeaway is that questions about the Hilbert function of fatpoints on $\mathbb{P}^{2}$ translate into questions about powers of linear forms in three variables, which are exactly the ideals $J(v)$ associated to the vertices of a tetrahedral complex. In particular, even for generic points (hence, for general linear forms), the form of the Hilbert function is unknown. Computing the constant term of the Hilbert polynomial for a generic tetrahedral complex would solve Conjecture 5.9. As long as there are eight or fewer planes, the corresponding linear system is anticanonical, and all is well. But even for $r=2$ it is possible to have nine distinct planes meeting at a point $v$, so $J(v)=\left\langle l_{1}^{3}, \ldots, l_{9}^{3}\right\rangle$. Since $\operatorname{dim}\langle x, y, z\rangle_{3}^{3}=10, J(v) \neq\langle x, y, z\rangle^{3}$, and also $J(v)$ does not correspond to an anticanonical linear system, so there is no easy way to compute the dimension.

\section{Open questions}

We close with a number of open questions. The most well known open conjecture is the dimension of $S_{3}^{1}(\Delta)$ when $\Delta$ is planar, and the generalization of this as Conjecture 1.2. We mention some additional interesting questions.

6.1. Higher dimensions. Reconcile the results of the last section with the results of Alfeld-Schumaker-Whiteley [7] on the dimension of $S_{d}^{1}(\Delta)$ for generic tetrahedral complexes and $d \geq 8$. Since $r=1$, as soon as there are six or more distinct planes adjacent to each vertex $v, J(v)=(x, y, z)^{2}$; this is analogous to the fact that in the $r=1$ planar case, unless there are only two slopes at $v$, then $J(v)=(x, y)^{2}$. The result of [7] on $S_{d}^{1}(\Delta)$ for $d \geq 8$ is equivalent to the vanishing of $H_{2}(\mathcal{R} / \mathcal{J})$ and $H_{1}(\mathcal{R} / \mathcal{J})$ in degrees $\geq 7$. It seems possible that in any dimension, if $r=1$ and $\Delta$ is generic, then $H_{i}(\mathcal{R} / \mathcal{J})_{d}=0$ for $d \gg 0$ and $i<k$. Proving this would yield a combinatorial formula for the dimension of $S_{d}^{1}(\Delta)$ for $d \gg 0$. Alfeld [3] has relevant results for the general case, and Alfeld-Schumaker [6] have results for $k=3$.

A second interesting question here is if there are higher dimensional analogs of the "crosscut" partition found in [11], and the pseudoboundary partitions studied in [40]. This would give special classes of subdivision where $H_{i}(\mathcal{R} / \mathcal{J})_{d}=0$ for all $d>i$, and so by Theorem 4.18, all dimension computations come down to 
understanding the $R / J(\tau)$ for all faces. As we saw in $\S 5$, for $k \geq 3$ this is nontrivial. More generally, find formulas for special configurations, as in [36] and [37].

6.2. Polyhedral complexes. In [18], Dipasquale proves that for a planar polyhedral complex $P$, if $F=\max \{n \mid$ there is an n-gon in $P\}$, then the formula of Theorem 4.17 applies if $k \geq(2 F-1)(r+1)-1$, and makes the

Conjecture 6.1. Theorem 4.17 applies if $k \geq(F-1)(r+1)-1$.

This agrees with Conjecture 1.2 when $P$ is simplicial. In higher dimensions, an appropriate analog [35] of Theorem 4.17 gives the top three coefficients of the Hilbert polynomial, but just as in the simplicial case, the remaining coefficients will be extremely delicate.

6.3. Supersmoothness. There has been no attempt to use algebraic methods to study splines with supersmooth conditions, despite the fact that the supersmooth conditions may be encoded algebraically. So this area is ripe for exploration.

Acknowledgments: I thank the Mathematisches Forschungsinstitut Oberwolfach, my fellow organizers Larry Schumaker and Tanya Sorokina, and the participants for a wonderful and stimulating workshop, Lou Billera for introducing me to the topic, and Mike Stillman for some of the best fun of my mathematical life. Macaulay2 [22] computations were essential to this work.

\section{REFERENCES}

[1] J. Alexander and A. Hirschowitz, Polynomial interpolation in several variables, Journal of Algebraic Geometry, 4 (1995), 201-222.

[2] J. Alexander and A. Hirschowitz, La methode d'Horace eclatee: application a l'interpolation en degre quatre, Inventiones Mathematicae 107 (1992), 585-602.

[3] P. Alfeld, Upper and lower bounds on the dimension of multivariate spline spaces, SIAM J. Numer. Anal., 33 (1996), 571-588.

[4] P. Alfeld, L. Schumaker, The dimension of bivariate spline spaces of smoothness $r$ for degree $d \geq 4 r+1$, Constr. Approx. 3 (1987), 189-197.

[5] P. Alfeld, L. Schumaker, On the dimension of bivariate spline spaces of smoothness $r$ and degree $d=3 r+1$, Numer. Math., 57 (1990), 651-661.

[6] P. Alfeld, L. Schumaker, Bounds on the dimensions of trivariate spline spaces. Adv. Comput. Math., 29 (2008), 315-335.

[7] P. Alfeld, L. Schumaker, and W. Whiteley. The generic dimension of the space of $C^{1}$ splines of degree $d \geq 8$ on tetrahedral decompositions. SIAM J. Numer. Anal., 30, 889-920, 1993.

[8] L. Billera, Homology of smooth splines: generic triangulations and a conjecture of Strang, Trans. Amer. Math. Soc., 310 (1988), 325-340.

[9] L. Billera, L. Rose, A dimension series for multivariate splines, Discrete Comput. Geom., 6 (1991), 107-128.

[10] L. Billera, L. Rose, Modules of piecewise polynomials and their freeness, Math. Zeit., 209 (1992), 485-497.

[11] C. Chui, R. Wang, Multivariate spline spaces, Journal of Mathematical Analysis and Applications, 94 (1983), 197-221.

[12] R. Courant. Variational methods for the solution of problems of equilibrium and vibration. Bulletin of the A.M.S., 49, 1-23, 1943.

[13] J. Dalbec, H. Schenck, On a conjecture of Rose, J. Pure Appl. Algebra, 165 (2001), 151-154.

[14] C. de Boor, A practical guide to splines, 2nd edition, Appl. Math. Sciences, vol. 27, Springer, Berlin-Heidelberg-New York, 2001.

[15] D. Diener, Instability in the spaces of bivariate piecewise polynomials of degree $2 r$ and smoothness order r, SIAM J. Numer. Anal., 27 (1990), 543-551.

[16] M. Dipasquale, Shellability and freeness of continuous splines, J. Pure Appl. Algebra, 216, (2012), 2519-2523. 
[17] M. Dipasquale, Lattice supported polyhedral splines, Adv. in Appl. Math. 55, (2014), 1-21.

[18] M. Dipasquale, Regularity of mixed spline spaces, preprint, arXiv 1411.2176, (2015).

[19] D. Eisenbud, Commutative Algebra with a view towards Algebraic Geometry, Graduate Texts in Mathematics, vol. 150, Springer, Berlin-Heidelberg-New York, 1995.

[20] J. Ensalem and A. Iarrobino, Inverse system of a symbolic power I, Journal of Algebra, 174 (1995), 1080-1090.

[21] A. Geramita, H. Schenck, Fat points, inverse systems, and piecewise polynomial functions, J. Algebra, 204 (1998), 116-128.

[22] D. Grayson, M. Stillman, Macaulay 2: a software system for algebraic geometry and commutative algebra, http://www.math.uiuc.edu/Macaulay2

[23] D. Hong, Spaces of bivariate spline functions over triangulation. Approx. Theory Appl. 7 (1991), 56-75.

[24] A. Iarrobino, Inverse system of a symbolic power III: thin algebras and fat points, Compositio Math., 108 (1997), 319-356.

[25] M. Lai, L. Schumaker, "Spline functions on triangulations" Cambridge University Press, London/New York, 2007.

[26] F.H.S. Macaulay, "The algebraic theory of modular systems," Cambridge University Press, London/New York, 1916.

[27] T. McDonald, H. Schenck, Piecewise polynomials on polyhedral complexes, Advances in Applied Mathematics, 42, (2009), 82-93.

[28] R. Miranda, Linear systems of plane curves, Notices Amer. Math. Soc., 46 (1999), 192-201.

[29] B. Mourrain, N. Villamizar, Homological techniques for the analysis of the dimension of triangular spline spaces, J. Symbolic Comput., 50 (2013), 564-577.

[30] L. Rose, Combinatorial and topological invariants of modules of piecewise polynomials, Adv. in Math., 116 (1995), 34-45.

[31] L. Rose, Graphs, syzygies, and multivariate splines, Disc. Comp. Geom., 32 (2004), 623-637.

[32] H. Schenck, Homological methods in the theory of splines, Ph.D. thesis, Cornell University, May 1997.

[33] H. Schenck, A spectral sequence for splines, Adv. in Appl. Math., 19 (1997), 183-199.

[34] H. Schenck, Computational Algebraic Geometry, Cambridge Univ. Press, Cambridge, 2003.

[35] H. Schenck, Equivariant Chow cohomology of nonsimplicial toric varieties, Transactions of the A.M.S., 364,(2012), 4041-4051.

[36] H. Schenck, Splines on the Alfeld split of a simplex and type A root systems, Journal of Approximation Theory, 182, (2014), 1-6.

[37] H. Schenck, T. Sorokina, Subdivisions of spline spaces, preprint, (2015).

[38] H. Schenck, P. Stiller, Cohomology vanishing and a problem in approximation theory, Manuscripta Math. 107 (2002), 43-58.

[39] H. Schenck, M. Stillman, A family of ideals of minimal regularity and the Hilbert series of $\mathcal{C}^{r}(\hat{\Delta})$, Adv. in Appl. Math., 19 (1997), 169-182.

[40] H. Schenck, M. Stillman, Local cohomology of bivariate splines, J. Pure Appl. Algebra 117 \& 118 (1997), 535-548.

[41] L. Schumaker, On the dimension of spaces of piecewise polynomials in two variables, Multivariate Approx. Theory, Birkhaüser (1979), 396-412.

[42] L. Schumaker, Bounds on the dimension of spaces of multivariate piecewise polynomials, Rocky Mountain J. Math. 14 (1984), 251-264.

[43] P. Stiller, Certain reflexive sheaves on $\mathbb{P}^{n}$ and a problem in approximation theory, Transactions of the A.M.S. $\mathbf{2 7 9}$ (1983), 125-142.

[44] G. Strang, The dimension of piecewise polynomial spaces and one sided approximation, Springer Lecture Notes in Computer Science, 365 (1974), 144-152.

[45] S. Tohaneanu, Smooth planar r-splines of degree 2r, J. Approx. Theory, 132 (2005), 72-76.

[46] W. Whiteley, A matrix for splines, in Progress in Approximation Theory, Academic Press, Boston, 1991.

[47] S. Yuzvinsky, Modules of splines on polyhedral complexes, Math. Zeit., 210 (1992), 245-254.

Schenck: Mathematics Department, University of Illinois Urbana-Champaign, UrBANA, IL 61801, USA

E-mail address: schenck@math.uiuc.edu 